\title{
Problemas planteados en la creación de índices de citas en el área de Humanidades: la base de datos ModernitasCitas y las publicaciones de Historia Moderna
}

\author{
Francisco Fernández Izquierdo*, Julia Osca-Lluch*, Mayte López **, \\ Juansa Sendra Roig ${ }^{\star \star \star}$, María Cruz Rubio Liniers ${ }^{\star \star \star \star}$
}

\begin{abstract}
Resumen: En este artículo se presentan algunos de los problemas encontrados en la extracción y homologación de referencias bibliográficas en una selección de revistas españolas de historia moderna y la creación de un índice de citas de revistas españolas de esta disciplina. Modernitascitas ha sido concebida como una base de datos para reunir y proveer información sobre la investigación española en historia moderna. Se presentan algunos datos sobre las dificultades y resultados obtenidos en la realización de un proyecto cuyo propósito central es servir de instrumento útil no sólo para los investigadores de esta disciplina sino también para los estudiosos y analistas de la ciencia, así como los generadores de datos estadísticos e indicadores de producción científica.
\end{abstract}

Palabras-clave: Historia moderna, referencias bibliográficas, índices de citas, revistas españolas, Modernitascitas.

\section{Problems encountered in the creation of a citation index in the humanities: The ModernitasCitas database and publications in Modern History}

Abstract: This article presents some of the problems encountered in the collection and standardization of references from selected journals of early modern Spanish history and the creation of an index of citations from Spanish journals of this discipline. Modernitascitas is a database designed to collect and provide information on Spanish research in modern history. Data are presented on the difficulties and achievements in carrying out a project whose main purpose is to provide a useful tool not only for researchers in this discipline but also for scientific scholars and analysts, as well as generating statistics and indicators of scientific production.

* Instituto de Historia, Centro de Ciencias Humanas y Sociales, CSIC. 28037 Madrid. Correo-e: fizquierdo@ih.csic.es.

** Instituto de Historia de la Ciencia y de la Medicina López Piñero (UV-CSIC). Valencia. Correoe: m.julia.osca@uv.es; mayte.lopez@uv.es.

*** Universidad Politécnica de Valencia. Dpto. de Sistemas Informáticos y Computación. Valencia. Correo-e: jsendra@dsic.upv.es.

**** Instituto de Estudios Documentales sobre Ciencia y Tecnología, Centro de Ciencias Humanas y Sociales, CSIC

Recibido: 4-6-09; aceptado: 28-4-10: 
Key-words: Early Modern history, Bibliographic references, Citation index, Spanish journals, Modernitascitas.

\section{Introducción}

Los estudios bibliométricos, nacidos en el ámbito de las ciencias de la naturaleza y de las ciencias aplicadas, también han llegado a las ciencias sociales y a las humanidades, pretendiendo ser un procedimiento objetivo para evaluar la calidad y la repercusión de las publicaciones científicas. Sin embargo, su uso todavía no está tan extendido entre las ciencias humanas y una parte significativa de las ciencias sociales. En humanidades la bibliometría se utiliza en forma puntual y esporádica y no de forma sistemática y planificada. Pese a las diferencias en sus objetos de estudio y la disparidad tanto en métodos como técnicas de trabajo, las ciencias y las humanidades comparten el hecho de que el conocimiento derivado de la investigación se difunde mediante publicaciones, que son utilizadas como material de referencia para nuevas investigaciones y nuevas publicaciones (Fernández Izquierdo, 2008).

El poco uso de los indicadores bibliométricos en humanidades se debe a la dificultad de obtener consensos sobre la determinación de la calidad de la investigación y del peso que han de tener las diferentes tipologías documentales. Además, es difícil encontrar fuentes de información que ofrezcan indicadores similares a los que están disponibles para las ciencias experimentales.

Los métodos y los indicadores bibliométricos empleados en las ciencias exactas y naturales, basados y monopolizados por el recuento de publicaciones en revistas científicas y en la citación como evidencia de la calidad, no se han mostrado tampoco eficaces en el ámbito de las humanidades. La razón principal radica en que las ciencias humanas poseen una serie de características cognitivas y unas prácticas de comunicación que las hacen diferentes del resto (Torres-Salinas y otros, 2009), entre ellas la Historia (Giménez-Toledo y Rubio-Liniers, 2009), tales como publicar y citar preferentemente libros y monografías (Lindhom-Romantschuk y Warner, 2002; Torres-Salinas y Moed, 2009; Rey Castelao, 2009), la orientación nacional de la investigación y los diferentes hábitos de citación y referenciación (Glänzel y Schoepflin, 1999). Otros autores consideran que el problema se encuentra en la mala cobertura de la Web of Science de la empresa Thomson Reuters (antes ISI), productora de las bases que se emplean universalmente en los estudios bibliométricos, porque presentan un gran sesgo hacia las revistas anglosajonas y escritas en inglés y donde solo el 18\% de las revistas indizadas son de humanidades, sin que ello se corrija por el momento en sus competidores, las bases de datos Scopus o Google Scholar.

La medición del impacto de las publicaciones científicas, en general, y de las revistas científicas, en particular, se ha convertido en el instrumento por excelencia para determinar la difusión y visibilidad, la relevancia e importancia y, subsidiariamente, la calidad de la actividad investigadora (Maltrás, 2003; Jiménez y 
otros, 2003). Para fijar el impacto de una publicación se requiere la construcción de bases de datos que, de una parte, registren los trabajos publicados en las principales revistas de los distintos campos del saber y, de otra, permitan contabilizar las citas que dichos trabajos puedan recibir.

El impacto de las revistas se venía analizando por ISI en Journal Citation Report (JCR del SCI y JCR del SSCI), a lo que hay que sumar nuevos indicadores recientemente introducidos tanto en WoS como en Scopus (Torres-Salinas y Jiménez Contreras, 2010). En nuestro país, la extraordinaria complejidad técnica que implica la creación de índices de este tipo junto a los elevados costes económicos que supone esta empresa (Osca-Lluch y otros, 2008), ha impedido, hasta hace poco, desarrollar nuevas bases de datos que sirvan de complemento a los productos de Thomson-Reuters, sin que la presencia observada recientemente de un mayor número de revistas españolas en Web of Science resuelva la situación (Rodríguez Yunta, 2010). Dejando aparte el trabajo de grupos que abordan esta problemática de forma general, como es Scimago (2006), dentro del área de ciencias sociales y humanidades, hay que destacar la creación de dos bases multidisciplinares, In-RECS (Índice de Impacto de las Revistas Españolas de Ciencias Sociales) elaborada por el grupo EC $^{3}$ Evaluación de la ciencia y de la comunicación científica de la Universidad de Granada y Resh (Revistas Españolas de Ciencias Sociales y Humanidades), por el Instituto de Estudios Documentales sobre la Ciencia y la Tecnología - IEDCYT (CSIC), a las que se suma la específica IN-RECJ (Índice de impacto de las revistas españolas de Ciencias Jurídicas) elaborado por el grupo $\mathrm{EC}^{3}$.

De manera complementaria, también se han publicado estudios recientes sobre las publicaciones en el área de Historia en particular, a partir de su presencia en bases de datos y OPAC y de la información interna de los consejos editoriales (García y Román, 1998; Malalana, 2007a y b; Malalana y otros, 2007), y otros puntuales relativos a las redes entre historiadores (Ortega, 2001). Dentro de esta misma disciplina, partiendo de experiencias previas en la creación y análisis de un índice de citas en revistas de Historia (Sanz y otros, 2002), se puso en marcha en 2004 la base de datos ModernitasCitas, elaborada por el Instituto de Historia del CSIC con la colaboración de investigadores de la Universidad Carlos III de Madrid, del Instituto de Estudios Documentales sobre Ciencia y Tecnología de Madrid (CSIC) y del Instituto de Historia de la Medicina y de la Ciencia López Piñero de Valencia (UV-CSIC).

\section{Objetivos de la base de datos Modernitascitas}

La base de datos Modernitascitas (http://www.moderna1.ih.csic.es/emc/) pretende ser un instrumento eficaz en el campo bibliográfico para los investigadores que permita conocer, no solamente la producción científica española en historia moderna, sino también las características de la actividad investigadora en esta disciplina, a partir de los hábitos de publicación de sus investigadores. Se selec-

Rev. Esp. Doc. Cient., 33, 3, julio-septiembre, 496-505, 2010. ISSN: 0210-0614. doi:10.3989/redc.2010.3.729 
cionó un grupo de 16 revistas universitarias y académicas de prestigio reconocido, y en su primera etapa (2003-2006) se incluyeron también actas de congresos publicadas en 2000 y 2001. La pretensión de exhaustividad en la extracción de referencias, contando con recursos limitados, hasta el momento ha permitido incorporar información publicada entre 2000 y 2006. La gran variedad de tipos documentales que consultan y citan los historiadores trae como consecuencia una gran diversidad de formatos de referencias bibliográficas, lo que impide emplearlas tal cual aparecen. Por otro lado, las prácticas y normas de citación aplicadas por los historiadores añaden otra dificultad, como consecuencia de que citan las referencias a los trabajos consultados a pie de página, mezcladas con fuentes manuscritas de archivo, explicaciones aclaratorias o información adicional. Ello obliga a analizar de forma cuidadosa todo el texto situado en el área de notas, con el fin de extraer las citas bibliográficas de las anotaciones o comentarios que suelen acompañarles.

Uno de los objetivos de este proyecto ha sido poner a punto un método automático para identificar y extraer las citas, así como para homologarlas, tarea que presenta una gran dificultad por la heterogeneidad en las formas de citación empleadas, la falta de rigor al citar que se observa en algunas publicaciones y la carencia de una base de datos de referencia (Fernández Izquierdo y otros, 2007).

\section{Control de calidad en la normalización de las citas}

Las referencias citadas extraídas se convierten en un formato etiquetado de intercambio bibliográfico, de donde pasan a una tabla de base de datos. Sin embargo, aún estamos lejos de poder utilizarlas para calcular indicadores o hacer estudios bibliométricos, pues falta homogeneizar todos los campos que vayan a emplearse para identificar y contabilizar información, comenzando por los nombres de los autores y responsables de las ediciones, así como los títulos de las revistas, obras colectivas y actas de congresos, además de las fechas de publicación, tipo de documento e idioma. El procedimiento ha consistido en:
a) eliminar las variaciones de caja y de signos de puntuación,
b) elaborar listas ordenadas de las variaciones y detectar equivalencias,
c) ensayar procedimientos automáticos para identificar variaciones "casi" idénticas,
d) bomologar dichas variaciones.

Unas breves cifras pueden ilustrar la magnitud del tratamiento necesario en una de las etapas del proyecto. En la fase inicial de este proyecto (2003-2006) se obtuvieron y homologaron 44.443 registros bibliográficos citados que fueron extraídos de 1.282 artículos y actas congresos publicados en 2000 y 2001. Tras la recogida de 9.379 nuevas citas durante 2007, en los primeros meses de 2008 
se procedió a revisar y homologar el contenido de los campos de autores y títulos de revistas/obras colectivas/actas de congresos, tanto en estas citas nuevas, como en las procedentes del proyecto anterior, se acumularon en una tabla de 54.822 referencias.

Autores. Para homologar los autores, tras elaborar las listas ordenadas de variantes y revisarlas de forma artesanal, se aplicaron 3.108 cambios en los nombres de autores principales y 1.643 en autores secundarios, cambios que afectaron especialmente a los últimos registros incorporados (9.379), pues eran los que no habían sido sometidos aún a normalización. Las cifras de los cambios las calcula el programa que realiza la sustitución global en la tabla, una vez establecidas las equivalencias de valores incorrectos con su valor correcto, y tras haber sido eliminadas de forma automática todas las variantes de caja (mayúscula-minúscula) también mediante programa. Las tablas con las equivalencias de variantes de autores tenían esta dimensión:

\section{TABLA 1}

\section{Cambios para bomologar los nombres de los autores citados}

\begin{tabular}{ll} 
Variantes de nombres de autores (muchas de ellas correspondientes al mismo autor): & 30.720 \\
Una vez homologados: & \\
$\mathrm{N}^{\circ}$ total de autores distintos en los 44.443 registros procedentes del proyecto anterior: & 15.854 \\
$\mathrm{~N}^{o}$ total de autores distintos en el fichero conjunto tras incorporar 9.379 citas: & 20.435 \\
\hline
\end{tabular}

Esto quiere decir que el aumento de 44.443 citas a $54.822(+23,5 \%)$ ha supuesto un aumento porcentual incluso mayor en el número de autores distintos, que han pasado de 15.854 a $20.435(+28,9 \%)$. Con esto se ofrece una idea de la enorme variabilidad que presenta la bibliografía citada, pues al incorporar nuevos registros siguen apareciendo nombres de autores nuevos, pese a que se intenten eliminar sus variantes. En este caso se han hecho revisiones sistemáticas en los nombres de los autores, homologándolos a su forma más completa, desarrollada y exacta, añadiendo segundos apellidos, poniendo nombres completos en lugar de iniciales, reponiendo sus acentos a los apellidos cuando los han perdido por la aplicación de mayúsculas o versalitas. Nuestro esfuerzo va justamente en contra de la tendencia hasta no hace mucho de dejar los nombres de los autores solo en mayúsculas y desprovistos de signos diacríticos. Es de sobra conocido que la informática permite con una sencilla función transformar un nombre de persona correctamente escrito a su versión solo en mayúsculas y sin acentos, pero la tarea inversa resulta mucho más compleja.

Revistas, obras colectivas, actas de congresos y tipo de documento. En menor medida que los autores, los títulos de revistas/obras colectivas/actas de congresos arrojaron para el conjunto indicado un incremento del 19,5\%, al pasar 
de 6.009 títulos diferentes a 7.181. Se procedió a elaborar las listas de variantes y a corregirlas. La asociación entre el contenido de este campo y el tipo de documento fue objeto de verificación. Se observó que, en una cierta medida, unas mismas obras citadas habían sido clasificadas en la variable tipodoc (= tipo documental) alternativamente como monografía, actas de congresos, obras colectivas o artículos. Para detectar, corregir y unificar tales discrepancias, además de para completar muchos títulos que habían sido citados de manera parcial o incorrecta, durante los meses de mayo y junio de 2008, M.C. Rubio Liniers, de nuestro equipo de investigación en el IEDCYT (CSIC), se ocupó de revisar la tabla general de las citas acumuladas. Muchas de ellas necesitaron cotejarse en catálogos en línea (OPAC) o en buscadores de Internet para averiguar su tipología documental, imposible de determinar con su mera citación, y también se eliminaron registros que se consideraron erróneos o innecesarios para el proyecto, lo que motivó alguna variación en los totales analizados respecto a lo dicho anteriormente respecto a los nombres de los autores.

Para medir el grado de esta corrección se comparó la distribución de tipos documentales asignados previa y posteriormente a esta revisión, y se observó que fueron necesarios más de 3.000 cambios (aprox. 1,5\% de registros erróneos en su tipo documental). La corrección supuso identificar especialmente actas y obras colectivas que habían sido consideradas inicialmente monografías. Igualmente, determinadas citas clasificadas como artículos de revista pasaron a clasificarse como obras colectivas y actas, gracias a la información ampliada por localización externa de los registros citados.

Aunque el esfuerzo fue considerable en la revisión artesanal de los más de 53.000 registros, a efectos numéricos globales un 1,5\% de error detectado supone un $98,5 \%$ de aciertos. ¿En qué medida afectaban estos errores a indicadores previamente calculados? En el año 2007 se hizo una presentación de los resultados iniciales del proyecto en el congreso de ISSI (Fernández Izquierdo y otros, 2007), y tras las correcciones comentadas arriba, aplicadas sobre las mismas citas procedentes de los años 2000 y 2001 — cuyo número disminuyó al detectarse duplicidades y otros problemas- se recoge en la tabla 3, con los tipos documentales más compactos que en la tabla 2.

Las correcciones aplicadas apenas han alterado la distribución general de los tipos documentales que se calculó inicialmente, con diferencias por tipos documentales que en ningún caso superan el punto porcentual (máxima diferencia observada "actas"). Igualmente, la variación relativa de algunos tipos documentales especiales (legislación, cartografía, soporte informático) apenas altera la distribución general. En el conjunto de las citas empleadas por los historiadores modernistas, las monografías siguen superando el 60\% del total, frente a los artículos de revista, situados en torno al 18\%. Tal distribución ha de matizar el valor real de los índices de impacto de las revistas españolas del área en el citado Resh, que se calcula solamente respecto a otras revistas españolas. En el caso analizado de la Historia Moderna, del 18\% que suponen las citas a revistas habría que sustraer las extranjeras, que según nuestros datos se sitúan en torno 
TABLA 2

Cambios en tipos documentales asignados a citas tras revisión global del fichero (mayo-junio 2008)

\begin{tabular}{|c|c|c|c|c|c|}
\hline Tipo de documento & $\begin{array}{c}\text { Frecuencia } \\
\text { antes de la } \\
\text { homologación }\end{array}$ & $\begin{array}{l}\% \text { tipo } \\
\text { doc. } \\
\text { antes }\end{array}$ & $\begin{array}{c}\text { Frecuencia } \\
\text { después de la } \\
\text { homologación }\end{array}$ & $\begin{array}{l}\text { \% tipo } \\
\text { doc. } \\
\text { después }\end{array}$ & $\begin{array}{c}\% \text { antes/ } \\
\text { después }\end{array}$ \\
\hline Actas & 3.130 & 5,82 & 3.327 & 6,18 & 5,92 \\
\hline Artículo & 9.903 & 18,40 & 9.527 & 17,70 & $-3,95$ \\
\hline artículo; actas & 134 & 0,25 & 149 & 0,28 & 10,07 \\
\hline artículo; fuentes & 120 & 0,22 & 46 & 0,09 & $-160,87$ \\
\hline Cartografía & 7 & 0,01 & 14 & 0,03 & 50,00 \\
\hline Legislación & 6 & 0,01 & 19 & 0,04 & 68,42 \\
\hline legislación; edición crítica & 51 & 0,09 & 65 & 0,12 & 21,54 \\
\hline legislación; fuentes & 163 & 0,30 & 189 & 0,35 & 13,76 \\
\hline Monografía & 24.684 & 45,87 & 23.886 & 44,39 & $-3,34$ \\
\hline monografía; capítulo & 322 & 0,60 & 699 & 1,30 & 53,93 \\
\hline monografía; edición crítica & 2.875 & 5,34 & 3.314 & 6,16 & 13,25 \\
\hline monografía; fuentes & 4.732 & 8,79 & 4.763 & 8,85 & 0,65 \\
\hline monografía; fuentes; web & 1 & 0,00 & 1 & 0,00 & 0,00 \\
\hline monografía; inédito & 20 & 0,04 & 18 & 0,03 & $-11,11$ \\
\hline monografía; inédito; fuentes & 121 & 0,22 & 121 & 0,22 & 0,00 \\
\hline obra colectiva & 6.592 & 12,25 & 6.612 & 12,29 & 0,30 \\
\hline Pintura & 2 & 0,00 & 2 & 0,00 & 0,00 \\
\hline Prensa & 127 & 0,24 & 130 & 0,24 & 2,31 \\
\hline prensa; fuentes & 223 & 0,41 & 288 & 0,54 & 22,57 \\
\hline Reseña & 27 & 0,05 & 27 & 0,05 & 0,00 \\
\hline reseña; fuentes & 1 & 0,00 & 1 & 0,00 & 0,00 \\
\hline reseña; prensa & 4 & 0,01 & 4 & 0,01 & 0,00 \\
\hline Revista & 28 & 0,05 & 45 & 0,08 & 37,78 \\
\hline soporte informático & 6 & 0,01 & 10 & 0,02 & 40,00 \\
\hline Tesis & 385 & 0,72 & 382 & 0,71 & $-0,79$ \\
\hline Web & 173 & 0,32 & 174 & 0,32 & 0,57 \\
\hline Total & 53.837 & 100,00 & 53.813 & 100,00 & \\
\hline
\end{tabular}


TABLA 3

Efecto de correcciones en el campo tipo de documento de la cita

\begin{tabular}{l|c|c|c|c|c}
\hline Tipo de documento & $\begin{array}{c}\text { Frecuencia } \\
\text { antes de la } \\
\text { homologación }\end{array}$ & $\begin{array}{c}\text { \% tipo } \\
\text { doc. antes }\end{array}$ & $\begin{array}{c}\text { Frecuencia } \\
\text { después de la } \\
\text { homologación }\end{array}$ & $\begin{array}{c}\text { \% tipo } \\
\text { doc. } \\
\text { después }\end{array}$ & $\begin{array}{c}\text { \% relativo } \\
\text { antes/ } \\
\text { después }\end{array}$ \\
\hline Monografía & 27.349 & 61,50 & 27.339 & 61,55 & $-0,04$ \\
\hline Artículos y reseñas & 8.286 & 18,63 & 7.923 & 17,84 & $-4,58$ \\
\hline Obra colectiva & 5.476 & 12,31 & 5.284 & 11,90 & $-3,63$ \\
\hline Actas & 2.464 & 5,54 & 2.903 & 6,54 & 15,12 \\
\hline Tesis & 321 & 0,72 & 320 & 0,72 & $-0,31$ \\
\hline Web & 168 & 0,38 & 170 & 0,38 & 1,18 \\
\hline Prensa & 217 & 0,49 & 220 & 0,50 & 1,36 \\
\hline Legislación & 177 & 0,40 & 235 & 0,53 & 24,68 \\
\hline Cartografía & 7 & 0,02 & 13 & 0,03 & 46,15 \\
\hline Soporte informático & 2 & 0,01 & 10 & 0,02 & 40,00 \\
\hline Pinturas & 44.473 & 100,00 & 44.419 & 100,00 & 0,00 \\
\hline Total & & & 2 & 0,00 & \\
\hline
\end{tabular}

al 23,6\% de los artículos citados, con lo que las revistas españolas representan en torno al 14\% del total de las publicaciones citadas. En la misma dirección, las repeticiones de cita a una misma obra, así como las citas a material de archivo merecen una atención que resulta inexistente en los estudios bibliométricos aplicados en campos ajenos a las humanidades.

Los títulos. Los títulos son comparados entre sí, para detectar ligeras variaciones que evitan la agrupación de indicadores a trabajos que son los mismos, aunque no aparecen citados de la misma forma. Las listas de valores controlados y homologados sirven además como índices que ayudan a las búsquedas en los sistemas informáticos, como puede comprobarse en la versión de consulta de ModernitasCitas en Internet y ayudan a detectar obras cuyo uso ha favorecido ediciones sucesivas.

\section{Discusión y resultados}

Los indicadores bibliométricos basados en publicaciones se han constituido en los últimos años como instrumentos útiles para estudiar los resultados de la actividad científica, pues aportan una valiosa información sobre la situación de la investigación y complementan con éxito al juicio de expertos (Bordons y Zulueta, 1999). Sin embargo, la fiabilidad de los estudios bibliométricos dependerá 
en gran medida del uso adecuado de los indicadores, y de las fuentes de información utilizadas. A diferencia de otras áreas científicas, en las humanidades, con independencia del peso que se pretenda dar al empleo de indicadores biblométricos entre otros métodos evaluadores de la calidad e impacto de la investigación y la difusión del conocimiento, existen importantes problemas materiales para disponer de índices equiparables a los de las bases de datos de citas de revistas. La base de datos Modernitascitas, basada en la producción española en historia moderna, supone una experiencia metodológica útil para otras ciencias humanas, y sus resultados informan con cifras inexistentes en otras fuentes sobre las características de la actividad investigadora en esta disciplina, a partir de los hábitos de publicación de sus investigadores.

\section{Agradecimientos}

La realización de este trabajo ha sido financiada por el Ministerio de Ciencia e Innovación a través de los proyectos HUM2006-13389-C03-02 y HUM200613389-C03-03

\section{Bibliografia}

Bordons, M., y Zulueta, M. A. (1999). Evaluación de la actividad científica a través de indicadores bibliométricos. Revista Española de Cardiología, 52, 790-800.

Fernández-Izquierdo, F.; Román-Román, A.; Rubio-Liniers, M. C.; Moreno-Díaz-del-Campo, F. J.; Martín-Moreno, C.; García-Zorita, C.; Lascurain-Sánchez, M. L.; García, P. E.; Povedano E., y Sanz-Casado, E. (2007). Bibliometric study of early modern history in Spain based on bibliographic references in national scientific journals and conference proceedings. Proceedings of ISSI 2007: 11th International Conference of the International Society for Scientometrics and Informetrics, vols I y II, 266-271.

Fernández Izquierdo, F. (2008). El pensamiento político hispánico en el contexto de la historiografía modernista: su presencia en las citas de publicaciones españolas de los años 2000 y 2001. En Aranda Pérez, F. J. y Damião Rodríguez, J. (eds.), De Re Publica Hispaniae. Una vindicación de la cultura política en los reinos ibéricos en la primera modernidad. Madrid: Editorial Sílex-Universidad de Castilla-La Mancha, 57-97.

García Marín, A. y Román Román, A. (1998). Las publicaciones periódicas de Historia Antigua, Prehistoria y Arqueología: difusión internacional. Trabajos de Prehistoria, 139-146.

Giménez-Toledo, E., y Rubio-Liniers, M. ${ }^{a}$ C. (2009). Características historiográficas y hábitos de publicación de los historiadores. Un ejemplo de las peculiaridades de las Ciencias Humanas en el marco de los sistemas de evaluación, en La documentación como servicio público. Estudios en Homenaje a Adelaida Román. CSIC, 107-129.

Glänzel, W., y Schoepflin, U. (1999). A bibliometric study of reference literature in the sciences and social sciences. Information Processing and Management, 35, 31-44.

Rev. Esp. Doc. Cient., 33, 3, julio-septiembre, 496-505, 2010. ISSN: 0210-0614. doi:10.3989/redc.2010.3.729 
Jiménez Contreras, E.; Moya Anegón, F., y Delgado López-Cozar, E. (2003). The evolution of research activity in Spain. The impact of the National Commission for the Evaluation of Research Activity (CNEAI), Research Policy, 32 (1), 123-142.

Lindhom-Romantschuk, Y., y Warner, J. (2002). The role of monographs in scholarly communication: an empirical study of philosophy, sociology and economics. Journal of Documentation, vol. 52 (4), 389-404.

Malalana Ureña, A. (2007a). Visibilidad internacional de las revistas españolas de historia medieval. En la España medieval, 455-496.

Malalana Ureña, A. (2007b). Visibilidad internacional de las revistas españolas de historia moderna. Cuadernos de Historia Moderna, 159-193.

Malalana Ureña, A.; Román Román, A., y Rubio Liniers, M. C. (2007). Visibilidad internacional de las revistas españolas de Historia. Scripta Nova: Revista electrónica de geografía y ciencias sociales, 11 (234).

Maltrás, B. (2003). Los indicadores bibliométricos: fundamentos y aplicación al análisis de la ciencia. Gijón, Trea.

Ortega Priego, J. L. (2001). Análisis de referencias basado en un modelo de espacios vectoriales: La investigación en Historia Contemporánea en Jaén durante 1990-1995. Revista Española de Documentación Científica, 369-381.

Osca-Lluch, J.; Velasco, E.; Blesa, P.; Barrueco, J. M., y Krichel, T. (2008). Some aspects of Citation Indexes in Spain. A comparative analysis. Scientometrics, 75 (2), 313-318.

Rey Castelao, O. (2009). El impacto de las políticas científicas en la investigación histórica reciente. XXXV Semana de Estudios Medievales. Estella, 21 a 25 de julio de 2008. Pamplona, 143-170.

Rodríguez Yunta, L. La alegría va por barrios (sobre Web of Science) (2010). Lista IWETEL, <http://listserv.rediris.es/cgi-bin/wa?A2=IWETEL;b8a08875.1004D> pub.2010-04-26 [consultado el 27 de abril de 2010].

Sanz, E.; Castro, F.; Povedano, E.; Hernández, A.; Martín, C.; Morillo-Velarde, J.; GarcíaZorita, C.; Nuez, J. L de la., y Fuentes, M. J. (2002). Creación de un índice de citas de revistas españolas de Humanidades para el estudio de la actividad investigadora de los científicos de estas disciplinas. Revista Española de Documentación Científica, 25 (4), 443-454.

Torres-Salinas, D., y Jiménez-Contreras, E. (2010). Introducción y estudio comparativo de los nuevos indicadores de citación sobre revistas científicas en Journal Citation Reports y Scopus. El Profesional de la Información, vol. 19 (2), 201-208.

Torres-Salinas, D.; Delgado López-Cózar, E., y Jiménez-Contreras, E. (2009). El análisis de la producción de la Universidad de Navarra en ciencias sociales y humanidades empleando rankings de revistas españolas y la Web of Science. Revista Española de Documentación Científica, vol. 32 (1), 22-39.

Torres-Salinas, D., y Moed, H. F. (2009). Library Catalog Analysis as a tool in studies of social sciences and humanities: An exploratory study of published book titles in Economics. Journal of Informetrics, 3 (1), 9-26. 